\title{
A PRECIPITAÇÃO dO ESTRÔNCIO NA FORMA dE CROMATO EM MEIO AMONIACAL E HIDROALCOOLICO *
}

R. A. CATAMI

J. T. PEREZ*

H. BERGAMIN FILHO

-E. S. A. "LUIZ DB QUBIROZ"

1. INTRODUÇÃo

A precipitaçāo do estrôncio na forma de cromato, em melo amoniacal e hidroalcoollico tem sido estudada por diversos autores (NOYES \& SWIFT, 1942; DAVIS, 1942; LELLAERT \& LECKHAUT, 1957). A citada precipitaçāo tem sido felta com a finalidade de caracterizar ou de separar o Ion $\mathrm{Sr}^{+2}$, mesmo em presença de $\mathrm{Ca}^{+2}$, com maior ou menor sucesso, de acôrdo com as condiçōes e o trabalho.

Por outro lado, KOLTHOFF, BELCHER e outros (1957), depois de isolarem o estrôncio na forma de cromato, determina ram aquêle cátion, indiretamente, através da titulação do croma to por iodometria.

Os autores do presente trabalho, procurando estudar um método volumétrico de determinar o estrôncio, em quantidades relativamente pequepas, verificaram que os dados obtidos, atra vés da técnica recomendada por KOLTHOFF, BELCHER e outros (1957) não eram satisfatórios. O método em questão consistia em precipitar o estrôncio pela adição de um número conhecido de equí valentes de $\mathrm{K}_{2} \mathrm{Cr}_{2} \mathrm{O}_{7}$, mas em excesso, e em meio amoniacal e hidroalcoolico. Depois de separado e precipitado, o excesso de dicromato era determinado na solução por lodometria, e o estrôn cio calculado.

Como os dados obtidos nāo estavam de acôrdo com as quantidades colocadas de estrôncio, julgou-se que seria mais acer tado isolar o precipitado e determinar o cromato no mesma. Entre tanto, os resultados óbtidos a partir do precipitado também náo

\footnotetext{
* Recebido pera publicsçlo en 8/8/62.

* Bolsista do Conselbo Nectonal de Pesgutses.
} 
estavam de acôrdo com as quantidades de estrôncio colocadas, o que sugeriu um estudo sobre a composiçāo do próprio precipitado, formado nas condiçöes descritas.

O presente trabalho tem como objetivo o estudo do com posto formado, quando se precipita o estrôncio com dicromato de potássio, em meio amoniacal e hidroalcoólico.

2. MÉTODOS

REATIVOS E SOLUGAO PADRAO:

SOLUÇAOO 0,100 NORMAL DE DICROMATO DE POTÁSSIO :

SOLUÇĀO 2 NORMAL DE CROMATO DE POTÁSSIO :

SOLUÇĀO DE HIDRÓXIDO DE AMÔNIO :

SOLUÇÃo DE ÁLCOOL A $95 \%$ :

SOLUÇÃO DE TIOSSULFATO DE SÓDIO 0,050 NORMAL :

SOLUÇÃO PADRÃO DE ESTRÔNCIO : dição de àcido clorídrico, p.a.
Foi preparada uma solução 0,100 normal de $\mathrm{K}_{2} \mathrm{Cr}_{2} \mathrm{O}_{7}$ a partir do sal sêco, p.a.

Foi preparada uma solução 2 nor mal de $\mathrm{K}_{2} \mathrm{Cr}_{2} \mathrm{O}_{7}$ a partir do sal sêco, p.a.

Empregou-se $\mathrm{NH}_{4} \mathrm{OH}$ p.a., $\mathrm{d}=$ 0,910 .

Fol preparada uma soluçāo con tendo $95 \%$ em volume de álco ol, a partir de álcool absoluto.

Esta soluçāo fol aferida com dicromato de potássio.

Preparada a partir de carbonato de estrôncio, p.a., que fol transformado em cloreto pela a

MÉTODO E MARCHA ANALITICA

a - Para se obter a relação entre a quantidade de 206 
estrôncio colocada e a de cromato determinada no precipitado, pro cedeu-se do seguinte modo:

Quantidades conhecidas de estrôncio (solução padrão) fo ram colocadas em tubos de centrifuga. Em seguida, foram adicio nados $15 \mathrm{ml}$ de solução 0,100 normal de $\mathrm{K}_{2} \mathrm{Cr}_{2} \mathrm{O}_{7}$ (como oxidan te), $1 \mathrm{ml}$ de $\mathrm{NH}_{4} \mathrm{OH}$ e um volume variável de água destilada de tal maneira que a soma dos volumes dos três líquidos permaneces se constante. Depois, foram acrescentados $15 \mathrm{ml}$ de solução hi droalcoólica (a $95 \%$ em álcool absoluto), agitando a solução. O pré cipitado foi deixado em repouso durante três horas e centrifugou-se a 3.000-3.200 rotaçōes por minuto, para a separação da fase sólida. Lavou-se o precipitado 3 a 4 vêzes, com solução hidroal coólica (com $95 \%$ de álcool absoluto) transferindo-o, em seguida, para um balāo de Erlenmeyer de $250 \mathrm{ml}$. O material sólido foi dis solvido com $15 \mathrm{ml}$ de soluçāo de $\mathrm{HCl}(1+4)$ e $\mathrm{o} \mathrm{Cr}_{2} \mathrm{O}_{7}{ }^{-2}$ foi dé terminado por iodometria, empregando-se solução 0,05 normal de $\mathrm{Na}_{2} \mathrm{~S}_{2} \mathrm{O}_{3}$.

Os dados obtidos estão no quadro 1, descrito em Resul tados Obtidos.

b - Para se obter o pêso do precipitado em função da quantidade de estrôncio colocada, procedeu-se assim :

Quantidades conhecidas de estrôncio (soluçāo padrāo) fo ram colocadas em tubos tarados de 50 ou $100 \mathrm{ml}$ da Centrífuga Internacional, tamanho 1, modêlo SBV. Foi adicionado um volu me variável de água destilada, de modo tal que somado ao da solu ção padrāo, o volume total permanecesse constante em tôdas as provas. Em seguida, foram adicionados 3 ou 6 gôtas de HCl $(1+9), 3$ ou $6 \mathrm{ml}$ de solução de $\mathrm{K}_{2} \mathrm{Cr}_{2} \mathrm{O}_{7}, 2$ normal, 15 ou 30 $\mathrm{ml}$ de soluçāo hidroalcoólica com $95 \%$ de ácool absoluto e 1,5 ou $3,0 \mathrm{ml}$ de $\mathrm{NH}_{4} \mathrm{OH}$. Após a adição de cada reativo, a solução fol homogenelzada. O dicromato em meio alcalino passou a cro mato e a precipitaçāo se processou. O precipitado fol deixado em repouso durante 2 horas e em seguida fol centrifugado. Lavou-se três vêzes, com solução hidroalcoólica com $95 \%$ de álcool, del xou-se secar em estufa a $809 \mathrm{C}$ durante.14 a 16 horas e pesou-se. 
Os dados obtidos encontram-se no quadro 2, em Resultados Ob tidos.

c - A análise qualitativa do precipitado foi executada pa ra os ions $\mathrm{NH}_{4}^{+}, \mathrm{K}^{+}$, alem do $\mathrm{Sr}^{+2}$ e $\mathrm{CrO}_{4}^{-\overline{2}}$. Para o $\mathrm{NH}_{4}{ }^{+}$empregou-se reativo de Nessler no destilado da solução do precipitado, tratado com hidróxido de sódio. Para o $\mathrm{K}^{+}$, foi usado o cobaltihexanitrito de sódio, após a eliminação do $\mathrm{NH}_{4}^{+}$.

d - A composiçāo provável do precipitado foi calculada, mediante a dissolução do precipitado e a determinação do estrôncio, amônio, potássio e cromato. O estrôncio foi determinado pela titulação do ácido oxálico procedente do oxalato, com permanganato de potássio. O amônio fol determinado pelo método comum de Kjeldahl. O potássio fol determinado pelo mé todo do cobaltihexanitrito (CATANI, 1954). Finalmente, o croma to foi determinado por iodometria, usando-se soluçāo de $\mathrm{Na}_{2} \mathrm{~S}_{2} \mathrm{O}_{3}$ 0,050 normal.

\section{RESULTADOS OBTIDOS E DISCUSSÃO}

Os dados obtidos referentes à relação entre a quantidade de estrôncio colocada e a de cromato determinada no precipitado são apresentados no quadro 1 .

\section{QUADRO 1}

RELAÇĀO ENTRE A QUANTIDADE DE ESTRÔNCIO COLOCADA, A DE CROMATO DETERMINADA E A DE ESTROONCIO CALCULADA.

\begin{tabular}{|c|c|c|c|}
\hline $\begin{array}{c}\text { mg de } \mathrm{Sr}^{+2} \\
\text { colocado }\end{array}$ & $\begin{array}{c}\text { ion miligrama de } \\
\mathrm{Sr}^{+2} \text { colocado }\end{array}$ & $\begin{array}{c}\text { ion miligrama de } \\
\mathrm{CrO}_{4}^{-2} \text { encontrado }\end{array}$ & $\begin{array}{c}\mathrm{mg} \text { de } \mathrm{Sr}^{+2} \\
\text { calculado }\end{array}$ \\
\hline 4,6 & 0,052 & 0,103 & 9,0 \\
4,6 & 0,052 & 0,100 & 8,8 \\
4,6 & 0,052 & 0,107 & 9,4 \\
9,2 & 0,104 & 0,206 & 18,0 \\
9,2 & 0,104 & 0,206 & 18,0 \\
9,2 & 0,104 & 0,210 & 18,5
\end{tabular}


$\mathrm{Na} 1 \mathrm{a}$. coluna do quadro 1 estão as quantidades em mili gramas de estrôncio colocadas. Na 2a. coluna, as mesmas quan tidades de $\mathrm{Sr}^{+2}$, expressas em lons miligramas. $\mathrm{Na} 3 a$. coluna, o número de ions miligramas de $\mathrm{CrO}_{4}{ }^{-4}$, calculado a partir da determinaçāo de $\mathrm{Cr}_{2} \mathrm{O}_{7}{ }^{-2}$ do precipitado, por iodometria. Final mente, na última coluna, a quantidade de estrôncio calculada, ad mitindo-se que o precipitado apresentasse a composição $\mathrm{SrCrO}_{4}$, isto $\dot{e}$, supondo-se um cromato de estrôncio simples, em que os ions estrôncio e cromato estivessem na proporçāo de $1: 1$.

Os dados obtidos sugeriram que a composição do precipi tado nāo pode ser representada simplesmente por $\mathrm{SrCrO}_{4}$, uma vez que a relação em ions miligramas foi bem diferente de 1 de $\mathrm{Sr}^{+2}$ para 1 de $\mathrm{CrO}_{4}^{-2}$. Realmente, a análise qualitativa do precipitado revelou a presença de quantidades apreclávels de $\mathrm{NH}_{4}^{+} \mathrm{K}^{+}$além de $\mathrm{Sr}^{+2}$ e de $\mathrm{CrO}_{4}{ }^{-2}$.

Uma vez verificado que o precipitado näo apresenta es trôncio e cromato, na proporção de 1:1 (expressa em lon- miligra ma) e que em sua composição entram também o potássio e o amô nio, procurou-se estabelecer a relação entre a quantidade de es trôncio e a do precipitado formado.

Os dados obtidos relativos ao pêso do precipitado em fun ção da quantidade de estrôncio colocada, acham-se no quadro 2 . (vide na página seguinte).

Os dados do quadro 2 evidenciam que a composiçāo do precipitado nāo pode ser representada simplesmente pelo $\mathrm{SrCrO}_{4}$. Assim, a última coluna do quadro 2 mostra que a relação entre o pêso do precipitado e o pêso do estrôncio colocado, variou de 4,50 a 4,90. Se o precipitado tívesse a composiçāo $\mathrm{SrCrO}_{4}$, a relação entre o pêso do precipitado e o pêso do estrôncio seria 2, 32. Não há dúvida portanto, que o precipitado apresenta uma composição mais complexa do que a apresentada pelo cromato de estrônclio comum.

Representando-se gräficamente a relaçāo entre o pêso de estrôncio (em miligramas) colocado e o pêso do precipitado em mi ligramas obtido, verifica-se que a citada relação é linear confor me mostra a figura 1. 
A equaçāo de regressão da reta OA da figura 1 é a se guinte :

$$
Y=4,58 X+0,84
$$

$\mathrm{X}$ è a variável independente e representa pêso em mili gramas, de estrôncio colocado.

$Y$ è a variável dependente e representa pêso em miligra mas, de precipitado obtido.

A fim de estabelecer a composiçāo provável do precipita do, dissolveu-se o mesmo em solução de $\mathrm{HCl}(1+9)$ e os ions $\mathrm{Sr}^{+2}, \mathrm{NH}_{4}^{+} ; \mathrm{K}^{+}$e $\mathrm{CrO}_{4}^{-2}$ foram determinados pelos métodos já descritos. Calculando-se a fórmula provável a partir dos da dos obtidos, chegou-se a seguinte composiçāo :

$$
6 \mathrm{SrCrO}_{4} \cdot\left(\mathrm{NH}_{4}\right)_{2} \mathrm{CrO}_{4} \cdot 6 \mathrm{~K}_{2} \mathrm{CrO}_{4}
$$

\section{QUADRO 2}

PÊSO DO PRECIPITADO OBTIDO PELA ADIÇĀO DE DICRO MATO DE POTÁSSIO, SOLUÇĀO HIDROALCOÓLICA E HIDRÓ XIDO DE AMÔNIO A QUANTIDADES VARIÁVEIS DE ESTRÔNCIO (VALORES MÉDIOS DE 3 REPETIÇŌES).

\begin{tabular}{|c|c|c|}
\hline $\begin{array}{c}\text { Pêso em mg de } \\
\text { estrôncio colocado. }\end{array}$ & $\begin{array}{c}\text { Pêso do precipitado } \\
\text { em mg (mêdia de } 3 \\
\text { valôres). }\end{array}$ & $\begin{array}{c}\text { Preparação entre o pếso } \\
\text { sodo precipitado e do } \\
\text { estrôncio colocado. }\end{array}$ \\
\hline 0,0 & 0,0 & - \\
1,8 & 8,3 & 4,61 \\
4,4 & 21,5 & 4,88 \\
8,8 & 43,1 & 4,90 \\
13,7 & 62,9 & 4,59 \\
27,5 & 126,7 & 4,61 \\
41,2 & 185,2 & 4,50 \\
50,5 & 235,3 & 4,66
\end{tabular}


Para comparar os dados obtidos referentes ao pêso do precipitado e à quantidade de $\mathrm{Cr}_{2} \mathrm{O}_{7}{ }^{-2}$, com os calculados, segun do a composição provável do composto:

$$
6 \mathrm{SrCrO}_{4} \quad\left(\mathrm{NH}_{4}\right)_{2} \mathrm{CrO}_{4} \cdot 6 \mathrm{~K}_{2} \mathrm{CrO}_{4},
$$

está sendo apresentado o quadro 3 .

\section{QUADRO 3}

COMPARAÇÃO DOS DADOS OBTIDOS REFERENTES AO PÊSO DO PRECIPITADO E À QUANTIDADE DE $\mathrm{Cr}_{2} \mathrm{O}_{7}{ }^{-2}$, COM OS CALCU U LADOS, SEGUNDO A COMPOSIÇĀO PROVĀVEL DO COMPOSTO :

$$
6 \mathrm{SrCrO}_{4} \cdot\left(\mathrm{NH}_{4}\right)_{2} \mathrm{CrO}_{4} \cdot 6 \mathrm{~K}_{2} \mathrm{CrO}_{4} \cdot
$$

\begin{tabular}{|c|c|c|c|c|}
\hline $\begin{array}{c}\text { Estróncio colocado } \\
\text { em miligramas }\end{array}$ & \multicolumn{2}{|c|}{$\begin{array}{c}\text { Pêso do precipitado } \\
\text { em miligramas }\end{array}$} & $\begin{array}{r}\text { Dicromato } \mathrm{Cr}_{2} \mathrm{O}_{7}{ }^{-2} \\
\text { em e. mg }\end{array}$ \\
\hline & obtido & calculado & obtido & calculado \\
\hline
\end{tabular}

$\begin{array}{rrrrr}1,8 & 8,3 & 8,7 & 0,130 & 0,133 \\ 4,4 & 21,5 & 21,3 & 0,326 & 0,326 \\ 8,8 & 43,1 & 42,5 & 0,648 & 0,653 \\ 13,7 & 62,9 & 66,1 & 0,950 & 1,017 \\ 27,5 & 126,7 & 132,8 & 1,975 & 2,041 \\ 41,2 & 185,2 & 199,0 & 2,975 & 3,057 \\ 50,5 & 235,3 & 243,9 & 3,435 & 3,747\end{array}$

$\mathrm{Na}$ 1a. coluna do quadro 3 são apresentados os dados re ferentes a quantidade de estrônclo colocada, em miligramas. Na 2a. e 3a. colunas, respectivamente, o pêso do precipitado obtido e o calculado, a partir da composiçāo provável:

$$
6 \mathrm{SrCrO}_{4}\left(\mathrm{NH}_{4}\right)_{2} \mathrm{CrO}_{4} \cdot 6 \mathrm{~K}_{2} \mathrm{CrO}_{4} .
$$

Na 4a. e na 5a. colunas, respectivamente acham-se 
apresentados os valôres, em equivalente-miligrama, de $\mathrm{Cr}_{2} \mathrm{O}_{7}{ }^{-2}$ determinado no precipitado e calculado a partir da composiçāo jä citada.

Como mostram os dados comparativos, há algumas dife renças relativamente grandes entre os resultados obtidos experi mentalmente e os calculados a partir da composiçāo provável do precipitado. Entretanto, devem ser levados em conta a existên cia de perdas do material, quer durante a lavagem (perdas mecâ nicas) como pela própria solubilidade na soluçāo hidroalcoólica que ainda não é conhecida.

De qualquer maneira, considera-se que o assunto mere ce ainda estudo, a fim de se confirmar a composiçāo do precipita do, estudo êsse que continua a ser executado.

Representando-se gràficamente, a relaçāo entre o pêso, em miligramas, do estrôncio colocado e o número de equivalentes-miligramas do dicromato determinado no precipitado, verifica-se que a citada relação é linear, conforme mostra a figura 2.

A equaçāo de regressão da reta $\mathrm{PB}$ da figura 2 è se guinte:

$$
\dot{Y}=0,0699 X+0,021
$$

$\mathrm{X}$ é a variável independente e representa pêso, em mili gramas, de estrôncio colocado.

$Y$ é a variável dependente e representa equivalente - mili grama de dicromato $\left(\mathrm{Cr}_{2} \mathrm{O}_{7}{ }^{-2}\right)$ determinado no precipitado.

A equaçāo (2) pode ser transformada em :

$$
X=\frac{Y-0,021}{0,0699}
$$

ou

$$
X=14,306(Y-0,021)
$$

A equação (3) poderia ser empregada para o cálculo de quantidade de estrôncio, a partir da determinação por iodometria, do número de equivalentes-miligramas de dicromato $\left(\mathrm{Cr}_{2} \mathrm{O}_{7}{ }^{-2}\right.$ ) procedente do precipitado. 
Na equaçāo (3), Y é o número de equivalente-miligrama de dicromato $\left(\mathrm{Cr}_{2} \mathrm{O}_{7}^{-2}\right)$ e $\mathrm{X}$ o número de milligramas de estrôn cio.

\section{CONCLUSÕES}

4.1. O precipitado que se obtem quando se adiciona di cromato de potássio, amoníaco e soluçāo hidroalco ólica com 95\% de álcool, a uma soluçāo contendo estrôncio, nāo corresponde ao cromato de estrôncio simples ( $\mathrm{SrCrO}_{4}$ ), mas a um cromato que, além de estrôncio, contém amônio e potássio.

4.2. A relação entre pêso de estrôncio colocado e pêso de precipitado obtido, no intervalo de 1,8 a $50,5 \mathrm{mg}$ de $\mathrm{Sr}^{+2}$, é dada pela equação de regressão:

Pêso de precipitado (em $\mathrm{mg}$ ) $=4,58$ pêso de estrôncio colocado $($ em $\mathrm{mg})+0,84$. O valor do coeficiente de regressão 4,58, es clarece que a composição do precipitado nāo pode ser dada pela foŕ mula $\mathrm{SrCrO}_{4}$, uma vez que a relação $\frac{\mathrm{SrCrO}_{4}}{\mathrm{Sr}}$ é igual a 2,32 .

4.3. A composiçāo provável do precipitado parece ser $6 \mathrm{SrCrO}_{4} \cdot\left(\mathrm{NH}_{4}\right)_{2} \mathrm{CrO}_{4} 6 \mathrm{~K}_{2} \mathrm{CrO}_{4}$ pelos dados obti dos através da analise quantitativa do mesmo.

4.4. A relaçāo obtida entre o pêso de estrônclo em milli gramas e o número de equivalentes-miligramas de dicromato $\left(\mathrm{Cr}_{2} \mathrm{O}_{7}{ }^{-2}\right)$ determinado no precipitado foi a seguinte: Pêso de estrôncio em milligramas $=14,306$. (número de equiva lentes-miligramas de dicromato - 0,021)

\section{RESUMO}

O presente trabalho relata os dados relativos a análise qualitativa e quantitativa do precipitado, que se forma quando se adiciona a uma soluçāo contendo estrôncio, dicromato de potássio, em meio amoniacal e hidroalcoólico. Em lugar de se formar cro mato de estrôncio simples, $\mathrm{SrCrO}_{4}$, forma-se um cromato que 
além do estrôncio, contém os lons amônio e potássio.

Soluçōes padrōes contendo desde 1,8 até $50,5 \mathrm{mg}$ de es trôncio, foram tratadas com soluçāo de dicromato de potássio $\overline{2}$ normal, amontaco e solução hidroalcoólica com $95 \%$ de álcool abso luto. O precipitado fol pesado e a equaçāo de regressão que rela clona o pêso do estrôncio colocado e o pêso do precipitado obtido, è a seguinte :

$$
\begin{aligned}
& Y=4,58 \mathrm{X}+0,84, \quad \text { onde : } \\
& X \text { é o pêso em mlligramas, do eatrônclo colocado } \\
& Y \text { é o pêso em mlligramas, do precipltado }
\end{aligned}
$$

A composiçāo provável do precipitado parece ser

$$
6 \mathrm{SrCrO}_{4}\left(\mathrm{NH}_{4}\right)_{2} \mathrm{CrO}_{4} \cdot 6 \mathrm{~K}_{2} \mathrm{CrO}_{4}
$$

\section{SUMMARY}

This paper describes the data obtained concerning qualita tive and quantitative analyses of the precipitate formed when to a solution containing strontium ion are added potassium dichro mate, ammonium hydroxdde and 95 per cent alcohol.

Three replications of standard solution containing 1.8 , $4.4,8.8,13.7,27.5,41.2$, and $50.5 \mathrm{mg}$ of strontium were treated with potassium dichromate and then, with constant stirring 95 per cent of alcohol and ammonium hydroxide. The precipitate was allowed to atend for two hours and afterward was centrifuged, washed with 95 per cent alcohol drled at 809C for 14-16 hours and weighed. Qualitative analysis of the precipitate showed potassium and ammonium ions, besides strontium and chromate.

The relationships between the weight of strontium and the weight of the precipitate obtained is given by the regression line ex pressed by the equation :

$$
Y=5.48+0,84 \text { where }
$$

$X$, is the weight of strontium in milligram

$Y$, is the weight of the precipitate in mflligram 
The value 4,58 for the $B$ parameter of the regression equation and the qualitative and quantitative analysis of the precip itate, suggested that its composition must be quite different from the simple strontium chromate $\mathrm{SrCrO}_{4}$. The data allow to admit the following probable composition :

$$
6 \mathrm{SrCrO}_{4} \cdot\left(\mathrm{NH}_{4}\right)_{2} \mathrm{CrO}_{4} \cdot 6 \mathrm{~K}_{2} \mathrm{CrO}_{4}
$$

\section{REFERÊNCIAS BIBLIOGRÁFICAS}

DAVIS, T. W. - Solubility of Strontium Chromate and the Detection of Strontium. Anal. Chem. 14: 709-711, 1942.

KOLTHOFF, I.M.; BELCHER, R. ; STENGER, V. A. \& MATSUYUMA, G. - Volumetric Analysis. New York, Interscience Publ. Inc., 1957. vol. II, pp. 337.

LELIAERT, G. BECKHAUT, J. - Investigation of Chemical Separation Methods for $\mathrm{Ca}$ and Sr. Anal. Chem. Acta. 16: $311-320,1957$.

NOYES, A. \&WIFT, E.H. - Qualitative Chemical Analysis of Inorganic Substances. $10^{\text {th }}$ edition. New York, The Mac Millan Co., 1942 . 



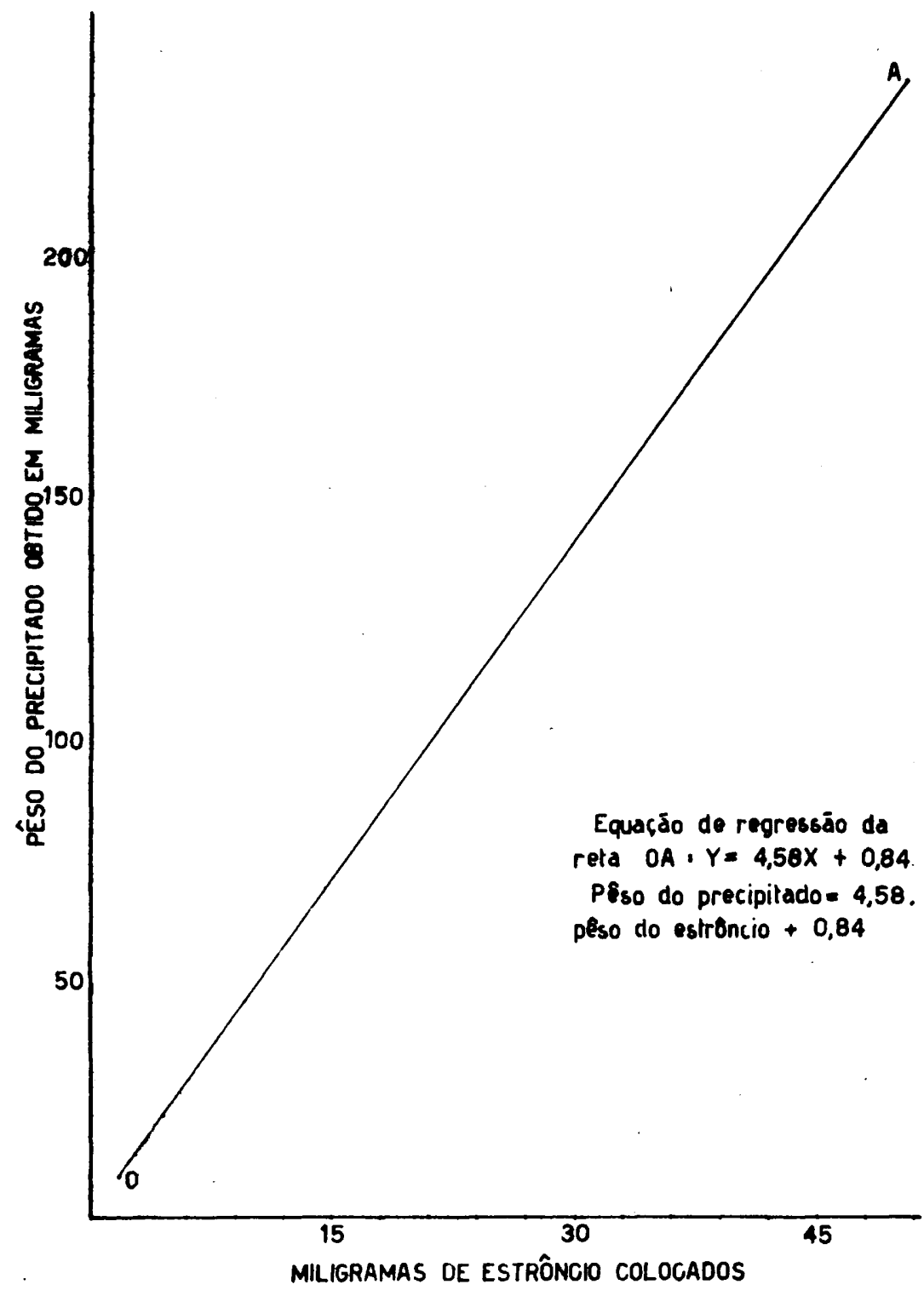

FIGURA 1- relaçăo entre o pêso de estrôncio colocadoc em miligramas) e $O$ pêso de precipitado obtido( em miligramas) 



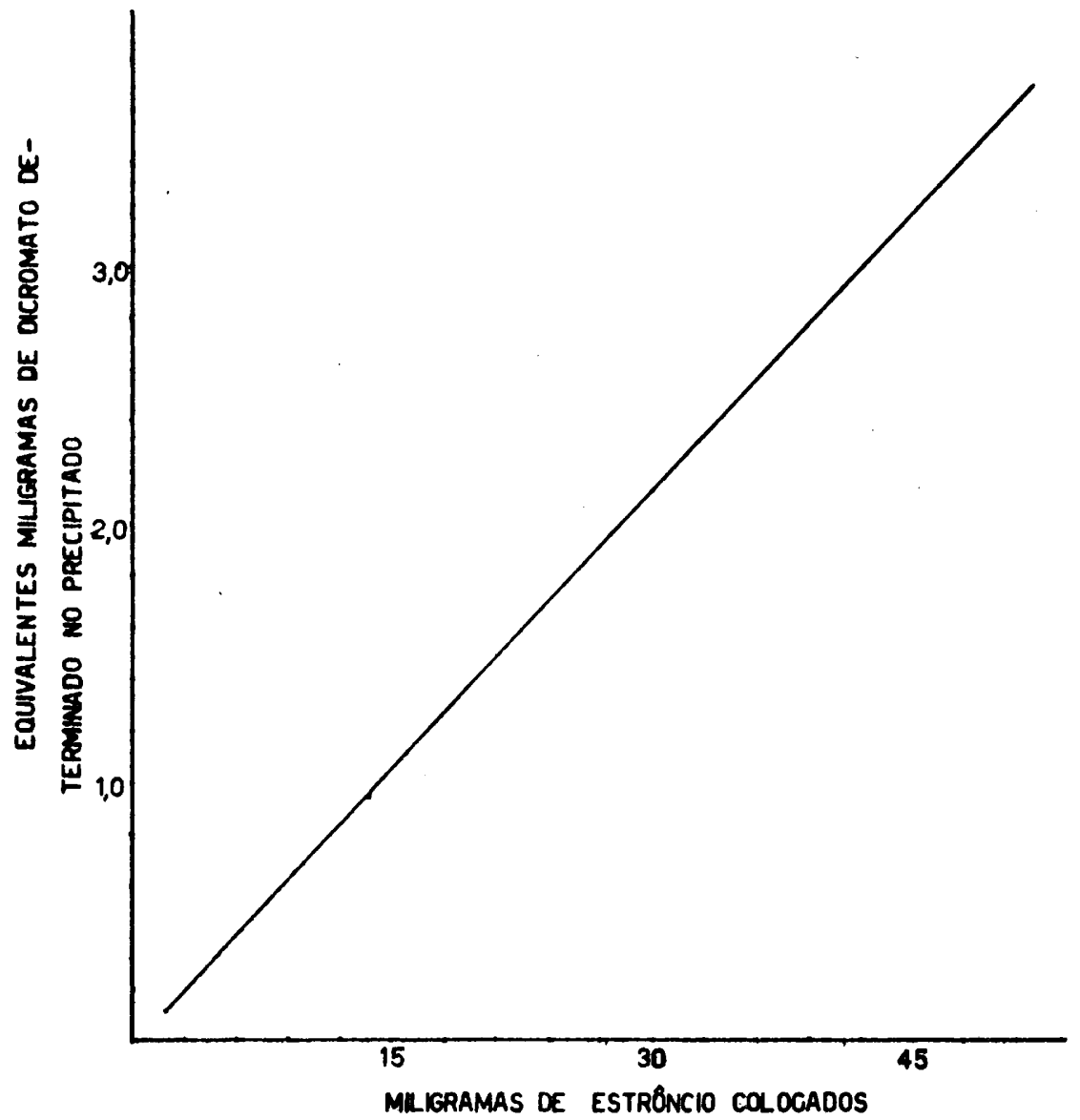

FIGURA 2- relação entre o pêso de estrôncio colocadoc em miligramass - o número de equivalentes-miligramas de dicromato determinado no precipilado 


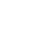

, 